

\section{State of the Field}

Structural geology and tectonics are core disciplines in the geological sciences, and play a central part in our study of the origin and evolution of solid planetary bodies. Structural geology focuses on the geometry, distribution, kinematics, dynamics, and mechanics of structures formed by rock and ice deformation; whereas tectonics pertains to the building of, and motions within, the uppermost deformable portions of solid surface planetary bodies $(1,2)$. Structural geology provides the fundamental tools to observe tectonic deformation across the Solar System, such that geologists are able to recognize and characterize structures and relate them to motions, stresses, and mechanical properties from the microscopic to the global scale.

Many planetary bodies across the Solar System preserve a record of deformation on their surfaces. Reflecting the widespread nature of tectonic deformation in planetary science, topics pertaining to structural geology and tectonics were rated as "somewhat" or "very relevant" among $83 \%$ of participants in a survey published in the Planetary Geologic Mapping_-Program Status and Future Needs open-file report (3). Planetary tectonics was also listed as a highly relevant field in the Challenges and Opportunities for Research in Tectonics community vision document submitted to the National Science Foundation in 2018 (4).

The study of planetary structural geology and tectonics is carried out with remotely sensed images, topography, and other data acquired from spacecraft flybys or from orbit, as well as more spatially limited in situ data from landers or rovers. But, fundamentally, the study of planetary tectonics benefits from, and requires the use of, the techniques and tools developed for Earth over the last two centuries (cf. 5).

Plate tectonics, a phenomenon where the uppermost portions of Earth's solid outer layer are mobile, interacting plates, is the paradigm that dominates geologists' thinking for our home planet. However, Earth-style plate tectonics is not recognized elsewhere in the Solar System. Other mechanisms have been invoked to account for tectonic deformation on planetary bodies with only a single, contiguous outer shell (sometimes called "one-plate planets"), and the structures and processes found on these bodies compare well with those in intra-plate settings on Earth. Such tectonic mechanisms can be local, regional, or global in nature and include impact cratering, vertical loading, volcanic/magmatic-tectonic interactions, mantle convective processes, or changes in planetary volume or figure caused by interior (thermal) or astronomical (rotational/tidal) factors.

\subsection{Rocky Bodies}

Virtually all rocky bodies we have visited or imaged in the Solar System show evidence for tectonic features. This finding is not surprising, given the myriad tectonic processes known to operate other than plate tectonics.

The range of tectonic structures on Earth is replicated elsewhere in the Solar System, from joints and other extensional fractures to normal faults, half-graben and graben, thrust faults, and strike-slip faults - although the latter structure is comparatively rare and is usually manifest as small ramps and tear faults in service of larger dip-slip structures. Folding is widespread, too, although is usually seen in association with thrust faults, such that hanging-wall anticlines are much more frequently documented than synclines. And, because only limited in situ outcrop-scale data are available (given the paucity of landers and rovers dispatched to planetary surfaces), many of the smaller tectonic structures 
familiar to Earth structural geology have yet to be documented (e.g., boudinage, foliations, etc.). Even so, the Opportunity and Curiosity rovers have imaged mineral veins on Mars, indicating that the same process of jointing and then mineral infilling by hydrothermal fluids so common on Earth also operated there, at least locally. Tectonic analyses have returned, among many such findings, that Mercury contracted radially by several kilometers over the past four billion years, manifest as huge systems of thrust faults with very limited extensional deformation (6); that graben and rift systems on Venus are abundant (7), with some of the oldest terrains, the tesserae, having tectonic fabrics implying that those units accreted (8); that many of the graben on the lunar nearside are underlain by dikes (9); and that the geometry of large thrust-faulted-related landforms on Mars can readily be used to estimate the architecture of those underlying faults (10), with implications for crustal structure and heat flow (11).

\subsection{I cy Bodies}

Icy satellites exhibit a multitude of tectonic structures that provide clues to the geological and orbital histories of these moons. For example, true polar wander (12) likely caused the outer shell of Enceladus to reorient, moving a low-density diapir and hotspot comprising from equatorial latitudes to the south pole (13). Jupiter's moon Europa exhibits evidence for non-synchronous rotation, where the icy shell has migrated with respect to a decoupled subsurface ocean (14), and plate-like motions that offer an opportunity to study tectonic deformation along plate margins in an icy lithosphere (15).

The orientations of structures reflected in the polygonal outlines of impact craters on Saturn's moon Dione indicate that this satellite may have experienced despinning and volume expansion during its history (16). For some moons, tectonism results from impact events or local endogenic resurfacing including diapirism, where lower-density material in the icy shell rises toward the surface (13). For example, the Uranian satellite Miranda exhibits highly deformed regions referred to as "coronae," which may have formed by diapirs as suggested by the analysis of bounding normal faults (17).

The surface geometries of large-scale faults, including evidence of proximal flexure, together with estimates of the rate of viscous relaxation of the icy shell, are important indicators of endogenic thermal properties of icy satellites (18). Heat fluxes estimated with these techniques are unexpectedly high in some cases, suggesting that orbital processes contribute energy that may have driven tectonic activity and possibly supported the presence of subsurface liquid water $(18,19)$. And the paucity of impact craters in some tectonized regions of icy moons indicates geological youth, and perhaps even ongoing tectonic activity. For example, Ganymede's ridged and grooved terrain (20), Miranda's coronae (21), Ariel's large chasmata, and much of Triton's surface also exhibit geologically young surfaces (22), highlighting the role of tectonics in icy body evolution.

\subsection{Minor Bodies}

Small bodies, including asteroids, comets, and irregular satellites show a remarkable variety of tectonic landforms, including pit crater chains (also referred to as "grooves"), troughs, polygonal impact craters, and ridges. Most of these landforms are the surface expressions of structures underneath regolith, with some large enough to be exposed at the surface - such as troughs on Vesta interpreted as grabens (23) and ridges on Eros regarded as thrust faults (24). Some impact craters on Eros have polygonal shapes, possibly controlled by pre-existing fractures within the target rock (24); similarly-shaped 
craters are also widely recognized on Ceres and Vesta $(254,26)$. Grooves are seen on Phobos (27) and on many asteroids, including Gaspra (28), Eros (24), Lutetia (29), and Vesta (30). These linear features are generally thought to be formed by collapse into a subsurface cavity driven by extensional deformation (31). Although these bodies are likely too small to have internally driven tectonic processes, impact events and tidal stresses may generate tectonic structures on regional and global scales. Impacts play an essential role by creating new fractures and reactivating existing structures, which in turn produce local and global fracture patterns depending on impact energy. Tidal stresses have been proposed to form global tectonic patterns such as the prominent grooves on Phobos that align with its direction of orbital motion (32), or the parallel sets of grooves on Saturn's moons Epimetheus and Pandora (33).

\subsection{Standard Structural Geology Methods}

The spectrum of planetary tectonics investigations all depend on those most fundamental of geological data products: maps. Structural maps convey the precise location and nature of tectonic structures, such as anticlinal hinges or graben walls using established symbology $(34,35)$. Image, topographic, and radar datasets, with a wide variety of properties (e.g., lighting conditions, resolution, etc.) are generally used as basemaps with which to identify and document landforms and structures. Linework associated with these features is drawn in geographic information systems $(3,36)$. Combined with lithological or morphological maps, structural mapping can highlight deformation patterns characteristic of specific rock units (37), inform models of subsurface structural architecture and formation mechanisms $(38,39)$, and support large-scale tectonic analyses to determine the timing and rate of tectonism and the directions of stresses or tectonic transport $(40,41)$.

Topography, images, and structural maps are commonly combined to numerically produce geometric, kinematic, and dynamic models of tectonic landforms that leverage aspects of the traditional structural method of geological profile construction and balancing. Because there are no seismic sections or borehole logs available as controls for numerical models as we use for such analyses on Earth, observed topography associated with tectonic landforms-assuming minimal erosion, especially on airless bodies - is an effective control for interpreting numerical model outputs. This approach is possible with a variety of applications including the USGS COULOMB code (9), or the Move Structural Modeling Software from Petroleum Experts, Inc. $(42,43)$.

Analogue modeling is also a widely employed research technique for understanding complex geological structures (44). Well-understood particulate simulants such as sand, glass beads, and clay act as counterparts to brittle geological materials, from a single basaltic volcano $(45,46)$ to an entire rocky or icy mechanical lithosphere $(47)$; viscoelastic and viscoplastic gels can simulate strain-dependent behavior in, for example, a shallow décollement or even a planet's mantle $(48,49)$. The relation of model to natural materials is achieved through the use of geometric, kinematic, and dynamic scaling ratios (50). Subject to the same physical laws as real-world geological processes, scaled analog models show remarkable morphological similitude to nature. Although physical modeling is under-utilized in planetary science, with preference commonly given to finite- and discrete-element modelling techniques, scaled analog models can be brought to bear on a range of planetary tectonics problems, from gravitational deformation, to regional crustal shortening, to ice shell expansion (47-49). 


\section{Outstanding Science Questions}

Numerous, major outstanding questions remain regarding the tectonic properties of rocky worlds throughout the Solar System. For instance, the early history of tectonic deformation of Mercury is unknown; was the period of global contraction there preceded by a phase of global expansion, akin to that which may have operated early in lunar history (51)? What of Venus' geological and tectonic history? With evidence for some tesserae having accreted, at least in some instances, what was the driving geological process, and is it still operating today? More broadly, does Venus show evidence for recent or even ongoing tectonic activity? Similarly, with marsquakes recently detected near faults (52), what can seismology teach us about the present-day rate and type of tectonics on the Red Planet?

Much is left to do regarding icy bodies, too. In particular, a key outstanding question is whether the configuration of structures on numerous icy satellites indicates the presence of a subsurface ocean and, if so, what can tectonic structures can tell us about the depth, age, and thermal properties of these oceans. And what of the icy shells themselves? It is not clear what caused the hemisphere-scale fault systems on many icy bodies, such as the complex and relatively young chasmata on the Uranian moon Ariel and the horst-and-graben structures that make up Ganymede's grooved terrain. It is also unclear if Saturn's moon Dione experienced an early or late expansion event during its history, and what implications such an early expansion would have for the presence and timing of a subsurface ocean within.

The science goals outlined by the Small Bodies Assessment Group (SBAG) regard tectonics as a key process for future exploration and investigation of minor bodies (53). Indeed, the deformation and tectonic characteristics of minor bodies are still not fully understood, especially in terms of how rock fracturing operates under low gravity. As many small bodies contain a thick regolith, how tectonic landforms relate to the types, sizes, and orientations of their subsurface structures has yet to be fully characterized. Other major questions include addressing how the surface expressions of structures reveal hidden tectonic patterns beneath the regolith, what processes form those structures, and what they tell us about the early history of the Solar System.

\section{Opportunities for Expansion Within the Field of Planetary Tectonics}

It is clear that a wide range of topics in planetary tectonics can be and are being addressed. However, there are also aspects and methods commonly applied to the study of structural geology and tectonics on Earth that are not yet widely utilized for topics in planetary tectonics.

For example, microtectonics deals with the characterization of structures on the microscopic scale, which yields abundant information on the history and type of deformation and in turn informs large-scale geological interpretations. Deformation can be inter- or intra-crystalline and involves pressure- or temperature-dependent deformation mechanisms, such as brittle fracturing or creep, respectively. Although this is a diverse and complex field for studying tectonized rocks of Earth, there is additional opportunity to study astromaterials, such as lunar samples or differentiated meteorites, from the perspective of microdeformation.

Future opportunities include the analysis of samples for microstructural textures or potential fluid inclusions (54), to learn about tectonic processes on their parent bodies. Such analysis could have far-reaching implications for early planetary evolution. Non- 
destructive geochemical analyses paired with X-ray microtomography, for instance, would reveal details on volatile speciation and their pathways of migration, thus placing better constraints on the types and mechanisms of volatiles feeding early atmospheres and/or oceans on planetary bodies. In addition, analysis of microstructure paired with geothermobarometry could provide tighter bounds for flow laws (i.e., temperaturedependent deformation), which often are the only means to estimate the strength-depth relationship of the deformable parts of planetary crusts and mantles. Future efforts to retrieve and study astromaterials could explicitly include these techniques for microstructural analyses.

Rock-mechanical testing in the laboratory is an effective means of enhancing our knowledge of tectonic deformation on planetary bodies (55), and may provide useful physical parameters for tectonic models of planetary bodies. There is a wide variety of deformation tests that can be carried out with specific boundary conditions imposed on the samples to simulate different planetary environments. Mimicking conditions unique to planetary settings has been used to understand the effects of temperature for deformation on Venus (56), for deformation of impact rock (57), and for faulting on icy bodies (58), but such studies are still relatively uncommon in the planetary literature.

Future experiments could employ triaxial compression tests to determine strength and elastic properties of intact and fractured materials using appropriate analogue samples and considering a variety of boundary conditions and apply them to settings previously not considered, such as the rocky seafloors of ocean worlds. Shear tests of planetaryanalogue fault gouge materials, fine-grained and usually unconsolidated rock that develops along faults, or fault planes in cold ice and icy mixtures will tell us of the frictional properties at the interfaces of two blocks of crust and the changes therein during the development of fault zones (59). These insights, in turn, will substantially improve our understanding of the seismic behavior of, and types of seismicity caused by, planetary faults, especially if considered in combination with seismic data (52).

Collecting geodesic and seismologic measurements would provide insights in present-day tectonic processes on planetary bodies. Seismometers and orbital geodesic methods, such as InSAR or GPS-equivalent spacecraft measurements, would detect quakes or geological changes that indicate to what extent and rate tectonics actively operate on planetary bodies.

Analogue structural fieldwork facilitates testing of hypotheses posed for planetary tectonic landforms, environments, and processes here on Earth via field research. Ideal tectonic analogue sites share rock types, stress field, or landforms with their planetary counterparts. Many field sites on Earth, especially in tectonically active regions of intraplate settings, in flood-volcanic provinces, or on ice shelves, are well-suited to enhance scientific discoveries. Structural relationships, timings and durations of deformation, or active tectonic processes can all be characterized in the field and used to better understand other planetary bodies. Fieldwork grounds planetary geologists, and helps build a framework for developing geologically realistic interpretations of tectonic landforms and processes. We therefore emphasize the importance of fieldwork for future planetary exploration and the education of future scientists involved in the analysis and interpretation of planetary structural geology and tectonics.

Advances in technology, computational methods, and 3D or virtual reality visualizations can advance research and be incorporated in teaching, adding to planetary fieldwork and increasing field accessibility to geoscientists. Subsurface 
geologic modeling that incorporates geostatistics and machine learning can lead to sophisticated geological models, and the growing body of open-source platforms (GemPy, Loop, Pynoddy) or visualization tools (ParaView, VRGS, QGIS) make such methods widely accessible to the planetary sciences (60).

4. Challenges to Increasing the Reach of Planetary Structural Geology and Tectonics

A relatively understudied aspect of planetary geology: Structural geology and tectonics together represent a substantial component of the geosciences discipline, but are largely focused on natural and mineral resource identification and exploitation, as well as on geotechnical hazards and civil engineering. Within planetary science, structural geology does not feature as widely as many other research fields. For example, of the almost 1,000 papers published in JGR Planets from 2010 through June 2020 , only $10 \%$ featured either "Structural Geology" or "Tectonophysics" as keywords (2\% and $8 \%$, respectively). Similarly, of the five Lunar and Planetary Science Conference programs published from 2016 through 2020, a total of 18 sessions focused on some element of structural or tectonic analyses of planetary bodies (broadly speaking, and encompassing geophysics), compared with 47 that had as their topics petrology, mineralogy, or geochemistry.

Limited graduate school opportunities: The Division for Planetary Science (DPS) webpage for graduate school opportunities in planetary science in the United States lists 53 highly detailed categories of planetary science, including geobiology or volcanology, but tectonics - arguably a field comparable to these categories_is missing from the list. Similarly, the GSA Planetary Geology Division website lists 59 universities with planetary geology programs, but only one specifically listing structural geology as a potential field of study. Students that are new to this field, but interested in the topic, face a challenge in identifying suitable programs across the US in which to study for an advanced degree in planetary structural geology and tectonics.

5. Enabling Future Discovery, Engagement, and Impact

There are opportunities to widen the scopes for further scientific discovery, welcoming additional scientific engagement, and increasing cross-disciplinary research for broadening the impact that the study of structural geology and tectonics can make in planetary science. We make the following recommendations for the planetary science community to consider:

$\rightarrow$ Broaden the scope of the research and encourage cross-disciplinary work. Expanding research in novel areas and building on advances in technology will facilitate highimpact science. Critical steps toward achieving this goal include more networking by holding dedicated, focused workshops and conference sessions.

$\rightarrow$ Making fieldwork more accessible for researchers of all career stages. Increasing the fieldwork opportunities, especially for undergraduate and graduate students to structural geology and tectonics research, will help to expand the reach of these techniques to planetary problems. Furthermore, increasing field accessibility to a wider geoscience community involves incorporating the wide range of possibilities of virtual field trips and fieldwork.

$\rightarrow$ Increase the visibility of current faculty and geoscience departments that offer an education and (graduate) degrees in planetary tectonics. Educating future generations of planetary scientists must include observation-driven photogeological interpretations of tectonic landforms on planetary bodies and how those features fit into our understanding of tectonic processes on Earth and beyond. 
$\rightarrow$ Increase tectonic techniques/viewpoints in active and future planetary exploration. The widespread occurrence of tectonic deformation across the Solar System necessitates that a thorough working of structural geology is essential to fully assess the science results from current and future planetary exploration, and so should factor early into relevant mission concept development, planetary data analysis, and collaboration.

$\rightarrow$ Support the Earth tectonics community in building a National Consortium for Tectonics (4). Engagement of scientists of the Earth- and planetary sciences in a joint initiative will help bridge gaps to strengthen research collaborations and facilitate future discovery.

\section{References}

[1] Fossen, H. (2016). Structural Geology, 2nd Edition. Cambridge University Press, 510pp. [2] Hatcher, R.D., Jr., Bailey, C.M., (2020). Structural Geology. Oxford University Press, 634pp. [3] Skinner, J.A., et al. (2019). Planetary geologic mapping-program status and future needs: USGS Open-File Report 2019-1012, 40pp. [4] Huntington, K.W., Klepeis, K.A. and 66 community contributors (2018). Challenges and opportunities for research in tectonics: A community vision document submitted to the U.S. National Science Foundation. University of Washington, 84pp. [5] Klimczak, C., et al. (2019). Principles of Structural Geology on the Rocky Planets. CJES 56, 1437-1457. [6] Byrne, P.K., et al. (2018). The tectonic character of Mercury. In: Mercury: The View after MESSENGER. Cambridge Planetary Science. [7] Solomon, S.C., et al. (1991). Venus tectonics: An overview of Magellan observations. JGR 97, 13199-13255. [8] Gilmore, M.S, Head, J.W. (2018). Morphology and deformational history of Tellus Regio, Venus: Evidence for assembly and collision. PSS 154, 5-20. [9] Klimczak, C. (2014). Geomorphology of Lunar Grabens Requires Igneous Dikes at Depth. Geology 42, 963-966. [10] Klimczak, C., et al. (2018). Topographic expressions of large thrust faults on Mars. JGR: Planets 123,1973-1995. [11] Mueller, K., et al. (2014). Fault and fold growth of the Amenthes uplift: Implications for Late Noachian crustal rheology and heat flow on Mars. EPSL 408, 100-109. [12] Matsuyama, I., Nimmo, F. (2008). Tectonic patterns on reoriented and despun planetary bodies. Icarus 195, 459-473. [13] Nimmo, F., Pappalardo R.T. (2006). Diapir-induced reorientation of Saturn's moon Enceladus. Nature 441, 614-616. [14] Kattenhorn, S.A. (2002). Nonsynchronous rotation evidence and fracture history in the Bright Plains region, Europa. Icarus 157, 490-506. [15] Kattenhorn, S.A., Prockter L.M. (2014). Evidence for subduction in the ice shell of Europa. Nature Geosci 7, 762-767. [16] Beddingfield, C.B., et al. (2014). Polygonal impact craters on Dione: Evidence for tectonic structures outside the wispy terrain. Icarus 274, 163-194. [17] Pappalardo, R.T., et al. (1997). Extensional tilt blocks on Miranda: Evidence for an upwelling origin of Arden Corona. JGR Planets 102, 13369-13379. [18] Peterson, G.F. et al. (2015). Elastic thickness and heat flux estimates for the uranian satellite Ariel. Icarus 250, 116-122. [19] Beddingfield, C.B., et al. (2015). Fault geometries on Uranus' satellite Miranda: Implications for internal structure and heat flow. Icarus 247. 35-52. [20 Pappalardo, R.T., et al. (2004). Geology of Ganymede. Jupiter: The Planet, Satellites and Magnetosphere, 363-396. [21] Beddingfield, C.B., Cartwright R.J. (2020). Hidden tectonism on Miranda's Elsinore Corona revealed by polygonal impact craters Icarus 343, 113687. [22] Zahnle. K. et al. (2003). Cratering rates in the outer Solar System. Icarus 163, no. 2. [23] Buczkowski. D.L. et al. (2012). Large-scale troughs on Vesta: A signature of planetary tectonics. GRL 39, L18205. [24] Prockter, L., et al. (2002). Surface expressions of structural features on Eros. Icarus 155, 75-93. [25] Neidhart, T. (2018). Polygonal impact craters (PICs) on Rhea, Dione, Tethys, Ceres and Vesta. PhD diss., Univ. Wien. [26] Hiesinger, H., et al. (2016). Cratering on Ceres: Implications for its crust and evolution. Science 353, 6303. [27] Basilevsky, A.T. et al. (2014). The surface geology and geomorphology of Phobos. PSS 102, 95-118. [28] Stooke, P.J. (1996). Linear features on asteroid 951 Gaspra. Earth, Moon, and Planets 74, 131-149. [29] Massironi, M., et al. (2012). Geological map and stratigraphy of asteroid 21 Lutetia." PSS 66, 125-136. [30] Buczkowski, D.L., et al. (2014). The unique geomorphology and physical properties of the Vestalia Terra plateau. Icarus 244, 89-103. [31] Smart, K.J., et al. (2011). Discrete element modeling of Martian pit crater formation in response to extensional fracturing and dilational normal faulting. JGR Planets 116, E4. [32] Hurford, T.A., et al. (2016). Tidal disruption of Phobos as the cause of surface fractures. JGR Planets 121, 1054-1065. [33] Morrison, S.J., et al. (2009). Grooves on small saturnian satellites and other objects: Characteristics and significance. Icarus 204, 262-270. [34] Crane, K.T., Klimczak, C. (2019). Tectonic Patterns of Shortening Landforms in Mercury's Northern Smooth Plains. Icarus 317, 66-80. [35] Callihan, M.B., Klimczak, C. (2019). Topographic expressions of lunar graben. Lithosphere, 11, 294-305. [36] Tanaka, K.L., et al. (2009). Planetary Geologic Mapping Handbook. Ann. Mtg. Planet. Geol. Map., San Antonio, TX.1-21. [37] Horgan, B.H., et al. (2020). The mineral diversity of Jezero crater: Evidence for possible lacustrine carbonates on Mars. Icarus, 339. [38] Grott, M., et al. (2007). Mechanical modeling of thrust faults in the Thaumasia region, Mars, and implications for the Noachian heat flux. Icarus, 186, 517-526. [39] Buczkowski, D.L., et al. (2012). Giant polygons and circular graben in western Utopia basin, Mars: Exploring possible formation mechanisms. JGR Planets, 117(E8). [40] Tanaka K.L., Davis P.A. (1988). Tectonic history of the Syria Planum province of Mars. JGR Solid Earth 93, 14893-14917. [41] Anderson R.C., et al. (2019). Unraveling the geologic and tectonic history of the Memnonia-Sirenum region of Mars: Implications on the early formation of the Tharsis rise. Icarus 332, 132-150. [42] Crane, K. (accepted manuscript) Structural interpretation of thrust fault-related landforms on Mercury using Earth analogue fault models, Geomorphology. [43] Herrero-Gil, A., et al. (2020). 3D modeling of planetary lobate scarps: The case of Ogygis Rupes, Mars. EPSL, 532. [44] Schellart, W. P., Strak, V. (2016). A review of analogue modelling of geodynamic processes: Approaches, scaling, materials and quantification, with an application to subduction experiments. $J$ Geodyn, 100, 7-32. [45] Oehler, J.-F., et al. (2005). Landslides and spreading of oceanic hot-spot and arc shield volcanoes on Low Strength Layers (LSLs): An analogue modeling approach. J Volcanol Geotherm Res 144, 169-189. [46] Sims, D. W., et al. (2013). Analog modeling of normal faulting above Middle East domes during regional extension. AAPG Bull, 97, 877-898. [47] Leonard, E.J., et al. (2019). Ridged plains reveal Europa's compressive past. LPSC 50, 2703. [48] Byrne, P. K., et al. (2013). A saggingspreading continuum of large volcano structure. Geology 41, 339-342. [49] Byrne, P.K., et al. (2015). Analogue modelling of volcano flank terrace formation on Mars. GSL Spec Pub 401. [50] Hubbert, M.K. (1937). Theory of scale models as applied to the study of geologic structures. GSA Bull 48, 1459-1520. [51] Andrews-Hanna, J.C., et al. (2013). Ancient Igneous Intrusions and Early Expansion of the Moon Revealed by GRAIL Gravity Gradiometry. Science 339, 675-678. [52] Giardini, D., et al. (2020). The seismicity of Mars. Nature Geoci 13, 205-212. [53] SBAG (2020), Goals and Objectives for the Exploration and Investigation of the Solar System's Small Bodies. ver. 2.0.2020, 44 pp. (http://www.Ipi.usra.edu/sbag/goals/). [54] Bodnar, R.J. (1999). Fluid inclusions in ALH 84001 and other Martian meteorites: Evidence for volatiles on Mars. Workshop on the Issue of Martian Meteorites 7002. [55] Heap, M.J., et al., (2017). Low surface gravitational acceleration of Mars results in a thick and weak lithosphere: Implications for topography, volcanism, and hydrology. Icarus, 281, 103-114. [56] Mackwell, S., et al. (1998). High-temperature deformation of dry diabase with application to tectonics on Venus. JGR 103, 957-984. [57] Heap, M.J., et al., (2020). Petrophysical properties, mechanical behaviour, and failure modes of impact melt-bearing breccia (suevite) from the Ries impact crater (Germany). Icarus, 349. [58] McCarthy, C. et al. (2012). Transient and steady state creep response of ice I and magnesium sulfate hydrate eutectic aggregates. JGR Planets 116, E04007. [59] Ikari, M.J., et al. (2011). On the relation between fault strength and frictional stability. Geology, 39, 83-86. [60] Wellmann, F. Caumon, G. (2018). 3-D Structural geological models: Concepts, methods, and uncertainties. Ad Geophys 59, 1-121. 